\title{
Precise Segmentation of Bone Marrow Cells by Using Multispectral Imaging Analysis Techniques
}

\author{
Qiongshui Wu* Libo Zeng Ningning Guo \\ Electronic Information School, Wuhan University, Wuhan 430079, China \\ * Corresponding author's E-mail: qswu@ whu.edu.cn
}

\begin{abstract}
Counting of different classes of white blood cells in bone marrow smears can provide pathologists with valuable information regarding various hematological disorders. For automatic imaging analysis techniques, precise segmentation of White Blood Cells is quite challenging due to the complex contents in bone marrow smears. Far more different from traditional color imaging analysis method, we introduced multispectral imaging techniques. After an image with high quality was acquired, the spectrum of each pixel was directly fed into a trained Support Vector Machine (SVM) for classification, then morphological binary operations were performed to correct the small error-classified regions. Masses of experiments show that our multispectral imaging method is device-independent to a large extent, and the segmentation results are highly satisfactory and inspiring. It proves that the introduction of multispectral imaging analysis techniques into White Blood Cells detection is a success. Multispectral imaging analysis is a promising technique in biomedicine.
\end{abstract}

Keywords: White blood cells; bone marrow image; multispectral imaging analysis; segmentation; SVM

\section{Introduction}

\subsection{Imaging analysis of bone marrow smears}

Counting of different classes of white blood cells in bone marrow smears can provide pathologists with valuable information regarding various hematological disorders. But it is tedious to manually locate, identify, and count these classes of cells, even by skilled hands. With the recent development of technology, much of this work can be done with the computer-assisted image analysis technology. Currently, the automatic bone marrow microscopic images analysis confronts significant difficulties due to: (1) The contents of this kind of images are so sophisticated, there are more than 20 different classes cells present[1]. (2) Image quality is significantly affected by the staining and illumination inconsistencies, which can lead to gray value variety, causing a big trouble for image segmentation. (3) The large variability of the white blood cells characteristics. The cells are frequently clustered, and there is no clear boundary between the nucleus and cytoplasm in many cases. These issues make precise segmentation a very challenging problem.

In the past decades, a lot of meaningful work had been done in bone marrow images segmentation[2-11]. Most of their work mainly focused on two difficult points: the segmentation of nuclei and the cytoplasm, along with cluster segmentation. This paper is focused on the former.

For the segmentation of nuclei and the cytoplasm, most of current work is based on color images. But for standard color image analysis, there are many problems[12]: Firstly, in the process of image acquisitions, the images are illuminant-dependent and whose qualities are influenced by spectral characteristics of the imaging system such as spectral responses of lens, optic inconformity and throughput properties, which make image reproduction very difficult. For example, identical samples may produce quite different image qualities even if they are imaged by the same type of instrument. So the robust of segmentation algorithms is very critical. Secondly, the standard digital RGB (Red, Green, Blue) values are the 
integrated results over the whole spectrum range, thus a lot of spectral information is inevitably lost, which means that a mass of chemical and physical information is hidden inside these spectral characteristics. While in many applications, cell detection, for instance, this amount of spectral information is very valuable for distinguishing slight color difference.
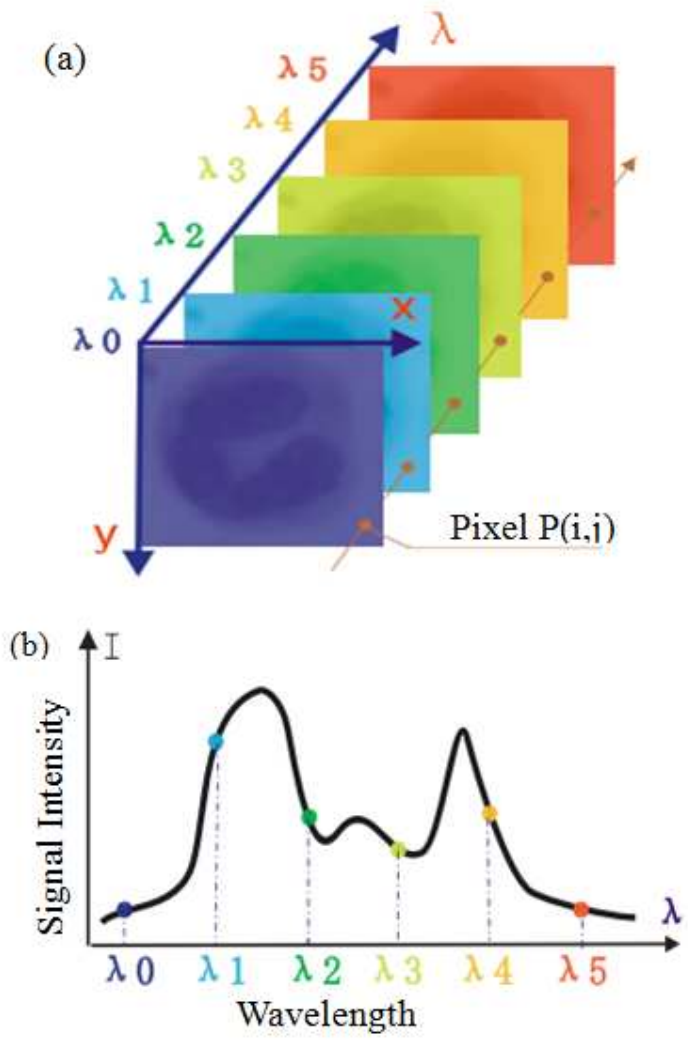

Figure 1 : (a) Multispectral image cube. (b) Spectrum at pixel $P(i, j)$.

In order to solve these problems and get better segmentation results, we introduced multispectral imaging[13-15] analysis techniques for automatic detection of White Blood Cells in bone marrow microscopic images. In this paper, a multispectral imaging microscope for bone marrow smears analysis is developed, and a spectral calibration algorithm is proposed for device-independent image acquisition, and then an approach of pixel-level classification based on spectral information was presented for precise segmentation, instead of traditional grey intensity or spatial one

Simultaneously recording spectral and spatial information of a sample, multispectral imaging, an innovative technology, has been widely used in remote sensing since the 80 's of last century. A multispectral image consists of a set of grey images, each acquired at a desired narrow band of wavelengths. As shown in Figure 1(a), the spectral image can be seen as a 3-dimensional image cube( $x-y-\lambda$ dimensions), the 2-dimensional image records the spatial information of a sample, and the third dimension represents spectral wavelength. Each pixel is associated with a spectrum. As shown in Figure 1 (b), by plotting the grey intensity of the images in the cube at the same position corresponding to wavelength sequently, the spectrum at this point could be obtained. Hence, a multispectral image can not only show the spatial features of the target, but also can reveal the unique spectral information of each pixel. From this point of view, spectral imaging is also called chemical imaging[16]. (a)
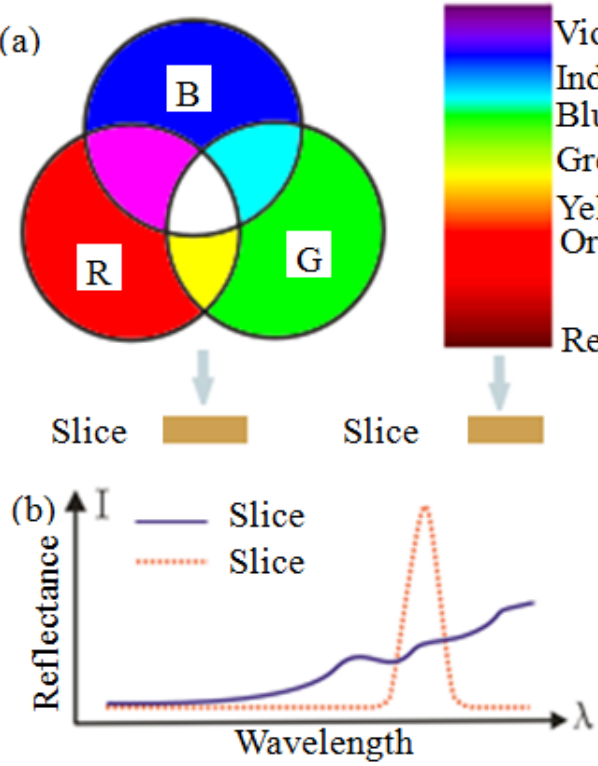

Figure 2: (a)Left panel: Color composition of RGB mode; Right panel: spectrum of white light. Slice (I) is formed by the RGB mode; Slice (II) is pure color extracted from the spectrum slice of white light. (b)Spectral Difference of color slice (I) and (II)

Figure 2 is a simple example to illustrate why spectral imaging is superior to traditional RGB model in color (chemical elements) differentiation. In Figure 2(a), slice (I) and slice (II) have no color difference in color to our naked eyes or color cameras. Actually, if analyzed by multispectral imaging method, we will find that they are quite different in spectrum (Figure 2(b)).

The combination of spectroscopy and image analysis technology can take advantage of various current tools applied in spatial image processing, chemometrics and specific spectroscopy, which makes it an ideal tool in studying the chemical states and physical properties of the samples. It is more powerful than spectral and image analysis or methodology taken alone. 


\section{Materials and Methods}

\subsection{Apparatus}

A schematic diagram of the multispectral imaging microscope apparatus is shown in Figure 3. This apparatus consists of a BX41 microscope (Olympus Corporation), a Liquid Crystal Tunable Filter (LCTF) device and its controller (VariSpecTM, Cambridge Research \&Instrumentation, Inc.), a three-dimensional automation stage, a Pentium IV computer (PC), a cooled monochrome Charge Coupled Device (CCD) camera (Penguin 600L, Pixera Corporation) and etc.

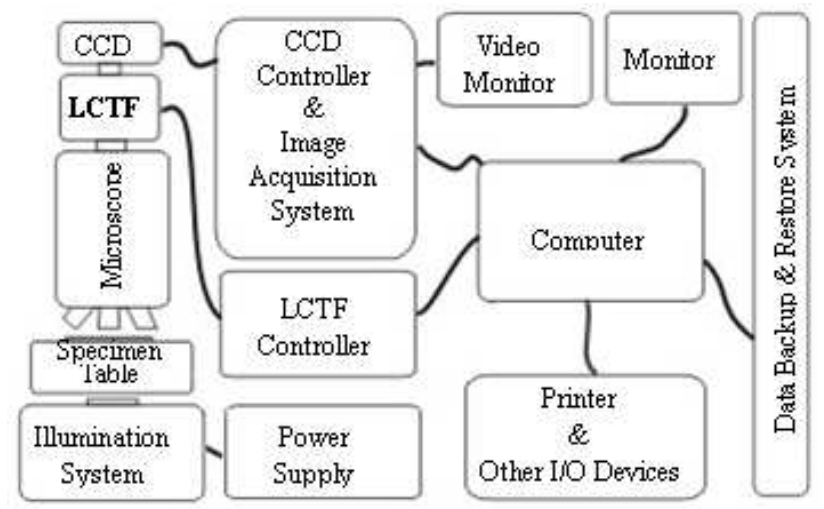

Figure 3: Schematic diagram of a multispectral imaging microscope apparatus based on LCTF

A 6V/30W halogen lamp is used as a bright-field source with Koehler illumination, which is powered by a stabilized power supply. A color-balancing filter is placed in the light path by attaching it to the filter mount of the microscope. The objective of $100 x / 1.3 \mathrm{NA}$ is used, and the condenser aperture iris diaphragm is set to $75 \%$ of the NA.

The LCTF device[17] is a tunable birefringent filter that can be used for both imaging and non-imaging spectral analysis. It is like a high-quality interference filter, but the wavelength of light is electronically controllable. It features solid-state with no moving parts, fast random access wavelength selection (switching time is approximate $60 \mathrm{~ms}$ ) and high image fidelity. The peak wavelength is electronically controlled by the computer via a RS-232 interface. The LCTF device is placed in the imaging path, one side coupled with a CCD Camera and the other side mounted on the microscope by the standard C-mount. The image signal of the sample is filtered by the LCTF. That is, the LCTF is used for wavelength selection.

The cooled monochrome CCD camera is with the resolution of $1024 \times 1024$ pixels and a dynamic range of 12 bits. It is used to detect the transmitted image that filtered by the LCTF.

A set of multispectral image processing and analysis software package was developed with Microsoft Visual C++ 6.0. It not only covers general image processing and analysis functions, but also has functions of multispectral analysis.

Bone marrow smears were prepared by Wright method, and provided by the Department of Hematological, Zhongnan Hospital of Wuhan University.

\subsection{Multispectral image acquisition method}

In order to take images at different wavelengths, the LCTF is used to provide wavelength selection range from $400 \mathrm{~nm}$ to $720 \mathrm{~nm}$ with an increment of $10 \mathrm{~nm}$. An image cube is acquired by continuously setting the filter to a desired wavelength, taking an image. This process is repeated over and over again until all the images under desired wavelengths are captured. All the grey-scale images of the same field of view, from the shortest to the longest wavelength, were stacked in a computer to create an "image cube", which is called multispectral image.

For a realistic imaging system, instruments with different optic imaging elements may cause different image quality. There are many factors that can significantly affect image quality, such as power of the lamp, optical spatial uniformity, and spectral characters of the whole system. The same segmentation algorithm may yield different results when imaged with different imaging environment. So, in order to decrease the dependence on image devices and make segmentation algorithm robust, the normalization of image is absolutely necessary.

Since the CCD, LCTF and optic element have their special spectral response, the whole sensitivity of the system is weak at both the longer and shorter wavelengths. In order to elevate the sensitivity at the weak response wavelength and improve image qualities, a clever CCD exposure control is used for spectral response compensation. The basic idea is, for poor sensitive wavelength, sets a longer exposure time. The exposure time determination method is described as following: Firstly, after the instrument has been powered on for about 15 minutes for stability, 33 grey images $B_{0}\left(i, j, \lambda_{k}\right)$

$((i, j)$ is the coordinates of a pixel in the image, $k=1,2, \cdots, 33)$ of a blank field view at the selected wavelengths $\lambda_{k}$ with the same exposure time $t_{0}$ are captured. And then, the mean grey value $M\left(\lambda_{k}\right)$ of the 33 images can be calculated out. Finally, the exposure time $t\left(\lambda_{k}\right)$ at wavelength $\lambda_{k}$ 
is determined:

$$
t\left(\lambda_{k}\right)=\min \left(t_{\max }, t_{0} * \frac{\eta}{M\left(\lambda_{k}\right)}\right)
$$

$t_{\max }$ is the maximum exposure time to limit $t\left(\lambda_{k}\right)$ in a settled range. $\eta$ is the maximum value of $M$.

After $t\left(\lambda_{k}\right)$ is determined, 33 grey images $B\left(i, j, \lambda_{k}\right)$ of a blank field view are captured at the calculated exposure time $t\left(\lambda_{k}\right)$ and saved as background images for later normalization use.

In a multispectral image acquisition process, the captured multispectral image $I\left(i, j, \lambda_{k}\right)$ with the exposure time $t\left(\lambda_{k}\right)$ are divided by the previously saved background images $B\left(i, j, \lambda_{k}\right)$ to generate the final normalized multispectral image $W\left(i, j, \lambda_{k}\right)$ :

$$
W\left(i, j, \lambda_{k}\right)=C \frac{I\left(i, j, \lambda_{k}\right)}{B\left(i, j, \lambda_{k}\right)}
$$

$C$ is a constant factor for rescaling the ratio image to a proper intensity that below 255(For 8-bit images, the maximum grey value is 255). In general, any value below 255 is proper. Here, we intuitively set $C$ the value 220 , which is about $85 \%$ of 255 .

\subsection{Segmentation}

\subsubsection{The goal of segmentation}

A bone marrow image mainly consists of three kinds of regions: nucleated cells, erythrocytes and background regions. The goal of segmentation is to find the nucleus and cytoplasm regions of nucleated cells.

\subsubsection{Segmentation method}

Figure 4(a) is a typical bone marrow image. From Figure 4(b), we can find that different regions have quite different spectra for this image, though whose colors may be very similar. So, we use spectral information other than traditional RGB color to distinguish different regions.

For each pixel $\mathrm{P}(\mathrm{i}, \mathrm{j})$ in a multispectral image, the spectrum (As shown in Figure 4 (b)) can be expressed as a vector $\mathrm{x}$ :

$$
x=\left(w_{\lambda_{1}}, \cdots, w_{\lambda_{n}}\right)^{T}
$$

$w_{\lambda_{k}}$ is grey intensity at wavelength $\lambda_{k}, n$ is the count of wavelength band, here, its value is 33 .

Our segmentation is a pixel level classification based on Support Vector Machines (SVM) [18-20]. The spectral vector of each pixel was directly fed into a $S V M$ classifier, and all the pixels were classified into four classes: nucleus, cytoplasm, erythrocytes and background.
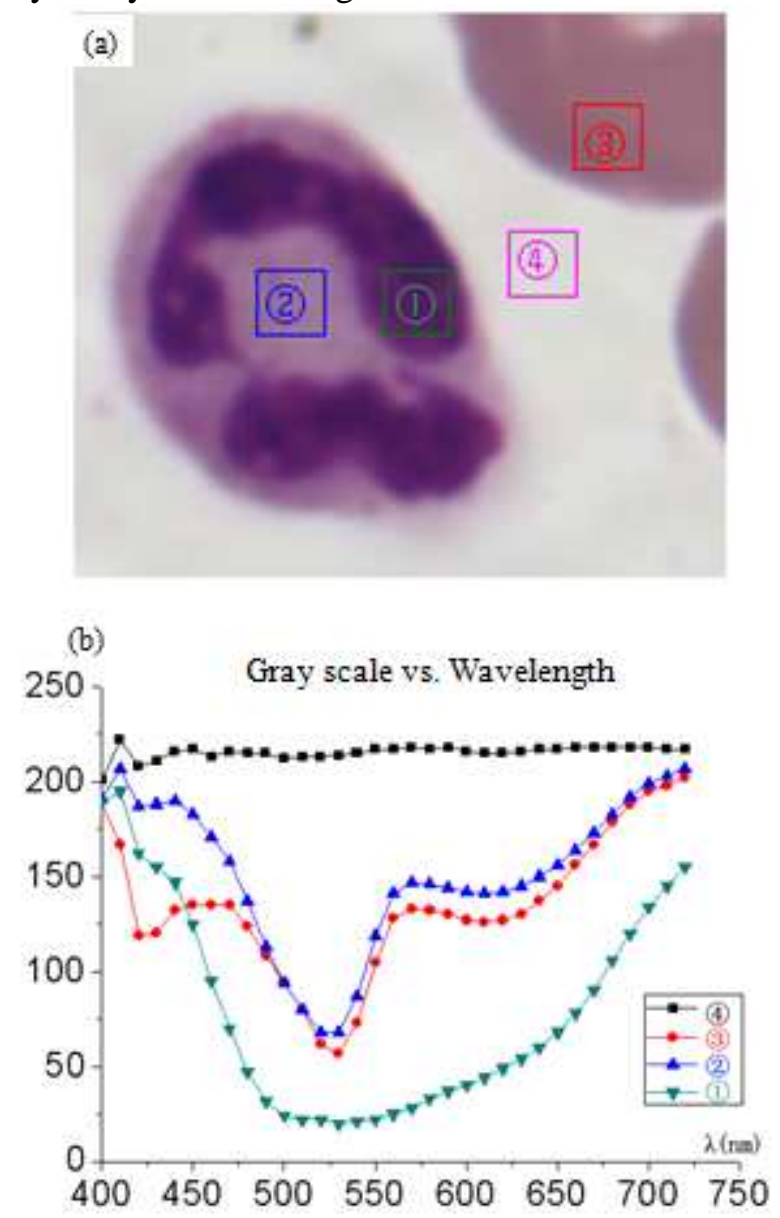

Figure 4: (a) A typical marrow bone image which consists of a polymorphonuclear leucocyte. (1)nucleus (2) cytoplasm (3)erythrocytes (4)background. (b)Transmitted spectrum of different parts marked in (a). Each rectangle region in (a) is regarded as a point, whose gray scale is the average in the rectangle.

$S V M$ is a new generation learning system based on recent advances in statistical learning theory. It delivers state-of-the-art performance in real-world applications such as text categorization, hand-written character recognition, image classification, biosequences analysis, etc.

$S V M$ has drawn much attention to Machine Learning, both theoretical and experimental. The remarkable generalization ability exhibited by $S V M$ can be explained through margin-based $V C$ theory. Its success in practice is due to its solid 
mathematical foundations: (1) Margin maximization. The classification boundary functions of $S V M$ maximize the margin, which corresponds to maximizing the generalization performance given a set of training data in machine learning theory. (2) Nonlinear transformation of the feature space using the kernel trick. SVM handles a nonlinear classification efficiently using the kernel trick which implicitly transforms the input space into another high dimensional feature space.

The SVM separates two classes with a hyperplane that maximizes the margin between the two classes of data. A set $S$ of $m$ training data can be represented by input-output pairs $\left(y_{1}, x_{1}\right), \ldots,\left(y_{m}, x_{m}\right)$ where each input data $x_{i} \in R^{N}$ belongs to a class $y_{i} \in\{-1,+1\}$ for $i=1, \ldots, m$. SVM formulates the optimal separating hyperplane between these two classes, which can be expressed as:

$$
g(x)=\sum_{i \in S V_{s}} a_{i} y_{i} K\left(x_{i}, x\right)+b
$$

where $a_{i}$ is a nonnegative Lagrange multiplier corresponding to $x_{i}, K($.$) is a kernel function$ and $b$ is the bias. The Lagrange multipliers are obtained as the solution of a convex quadratic programming problem. The data points $x_{i}$ 's corresponding to $a_{i}>0$ are called support vectors.

Such $x_{i}$ 's are the only data points in the training set relevant to classification since the decision surface is expressed by these points alone (support vectors). For linearly separable problem the number of support vectors and the hyperplane are determined by a subset of the training set only. For nonlinearly separable problem, $a_{i}$ in SVs is constrained by an upper bound $C$ which is regarded as a regularization parameter. This parameter makes a tradeoff between margin maximization and classification error.

The segmentation steps include two processes: training and classification.

The training process can be outlined below:

Thirty-five multispectral images(100x Objective) were manually segmented under the direction of a pathologist.

Many interested regions of nucleus, cytoplasm, erythrocytes and background (we label the different region classes with $1,2,3,4$ individually) were manually marked with different color mask according to their region classes. And then the spectrum of each pixel in the marked regions was extracted as feature vector, and its region class is used as class type. All the spectral features and class types were saved as standard training dataset.

When all the thirty images were completed, all the saved training dataset were fed into a $S V M$ classifier for training. There were totally 3,530 nuclear pixels, 6,399 cytoplasmic pixels, 1,696 erythrocytic pixels and 1,275 background pixels were saved as training sample sets. After training, the model results were obtained.

For tested image, the spectral vector of a pixel was fed into the trained classifier for classification, and the output of the classifier was the class type of the pixel.

C-support Vector Classification(C-SVC)[19,21] was used as a SVM classifier. Since the classification was a multi-class classification, we chose the one-against-one approach [22, 23] in which $\mathrm{k}(\mathrm{k}-1) / 2$ classifiers and each one trained data from two different classes were constructed. In classification a voting strategy was accepted: each binary classification was considered to be a voting where votes can be cast for all data points. Finally, each point was designated to be in a class with maximum number of votes. A polynomial was selected as the kernel function, and the degree of the polynomial was set to the value of 3 . The program code and more detailed discussions about $C$-SVC algorithm can be found at Dr. Chih-Jen Lin's Home Page[24].

Since $C-S V C$ is not very fast, if every pixel in a multispectral image is classified with $C$-SVC, it will be very time-consuming. In order to increase the classification speed, background regions were firstly removed by a constant threshold value of 200 to the grey image at wavelength $530 \mathrm{~nm}$. Figure 4(b) shows that the difference between background and other regions is the most significant, so a constant threshold is enough for the removal of background.

After segmented by the $C-S V C$, a series binary operations were applied to correct the small error-classified regions and make the edge of cell more smooth: Firstly, hole filling was performed to fill the small holes in the cell areas; Secondly, a watershed algorithm was performed to split the connected areas; Thirdly, small areas were deleted; Finally, binary open operation was performed to make the edge more smooth.

\section{Results and discussion}

\subsection{Performance of image acquisition}


The systematic nonlinearity calibration result is shown in Figure 5. Line (1) is the spectrum of a blank area, which is captured with the same CCD exposure time at all the different wavelengths. We can see that in the whole imaging system, the illuminant emission and spectral response is not conformity through the wavelength range, and the response is sensitive in the middle wavelength range while insensitive at both ends. Line (2) is formed by using the calculated exposure time at each individual wavelength. We can see that at each end, the response is enhanced. Line (3) is formed as equation (1) does, which is the real spectrum of blank areas in a multispectral image.

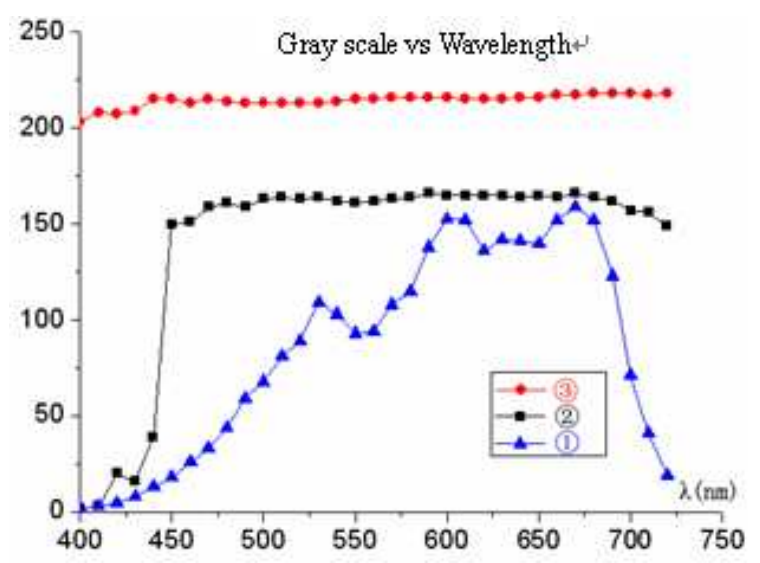

Figure 5: Background spectral calibration. (1)Spectrum of a blank area with same exposure time (2)Spectrum of a blank area with the calculated exposure time (3)Final spectrum of a blank area after calibration

\subsection{Segmentation Results}

One hundred and eighteen multispectral images other than the training sets acquired with $100 \mathrm{x}$ objective were used to evaluate the segmentation algorithms. There were 245 cells in these 118 images. Part of segmentation results are shown in Figure 6.

The segmentation results were evaluated by the satisfaction of a pathological expert. For all the 118 images, the segmentation of the background and erythrocytes were extremely well, there were very few errors. For the 245 cells, they can be segmented out $100 \%$. For nuclear segmentation, 235 cells were satisfactory. For cytoplasmic segmentation, 227 cells were satisfactory.

In all the cells, the correct ratio of acidophilic granulocytes and basophiles is not very high. One reason is that the color of the nucleus has a big variance, and another is that they occupy no more than 1 percent of all the cells, which makes enough training samples not available. For the cytoplasmic segmentation, the correct ratio of metarubricytes is not very high. This is due to their cytoplasmic color is quite similar to erythroblast, which were wrong segmented by erythroblast.

\section{Conclusions and future work}

From line (3) of Figure 5, we can see that for a blank area, the response at different wavelengths is almost the same. We can conclude that the imaging system was well calibrated and normalized, and the imaging method we used can decrease the dependence on imaging devices and imaging environment, which makes the later algorithms of image processing more robust.

A mass of experiments showed that our segmentation results are highly satisfactory. From Figure 6, we can conclude that the segmentation results are pretty good. Using spectral information for the detection of White Blood Cells is a successful exploration, and SVM-based classifier is also a success in spectrum classification.

Compared with traditional imaging analysis method, ours has following advantages: (1) Images are not so excessively dependent on imaging devices and imaging environment, which makes the later algorithm of image processing more robust. (2) Introduction of spectral information into segmentation makes the segmentation algorithm more popular and extensible. In traditional method, an effective algorithm for object detection may cause wrong results if the analyzed target changes. For example, if there is an algorithm very effective in red object segmentation, when used in blue object detection, the algorithm and parameters have to be modified, or it will not work correctly. In our method, however, it is not necessary to modify the algorithm , but to simply change the training samples. (3) Multispectral image contains more information than traditional color image, which makes the analysis method more flexible. For the later feature extraction or classification, it can provide more meaningful features, which is helpful for the improvement of classification accuracy.

Though preliminary results show that our multispectral imaging analysis method in bone marrow smears application is promising and inspiring, yet there is still a lot of room for improvement: (1) The wavelengths we used are range from $400 \mathrm{~nm}$ to $720 \mathrm{~nm}$ with an increment of $10 \mathrm{~nm}$, if a small increment is used, better results might be obtained. But it is really a time-consuming work to do so, and will occupy a tremendous disk 
space of our computers. (2) Boosting image acquisition and segmentation speed. Currently, its speed is not high enough for practical applications. But it is a new research in bone marrow image segmentation, and it is promising in biomedical image processing. (3) Focusing on lower magnification images (40x objective) analysis. One advantage is that it can increase the scan speed than with $100 x$ objective. Another is that no immersion oil is needed for $40 \mathrm{x}$ objective. A preliminary experiment shows that it is feasible and practical.
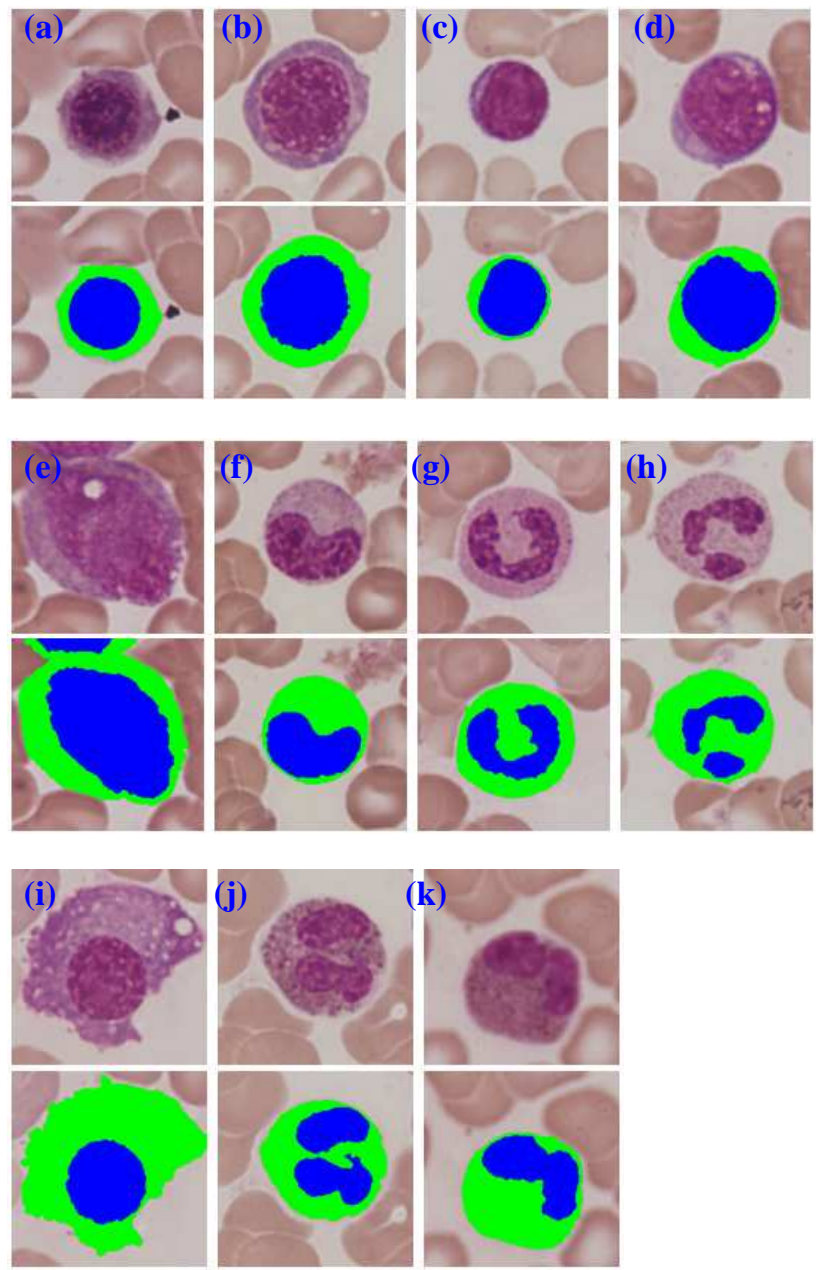

Figure 6: Segmentation results. The upper row is the original image and the next row is the segmented result. Blue color represents nuclear area and green color represents cytoplasmic area. (a) metarubricyte (b) polychromatic normoblast (c) lymphocyte (d) monocyte (e) neutrophilic myelocyte (f) neutrophilic metamyelocyte (g) band-cell (h) polymorphonuclear leucocyte (i) plasmocyte $(\mathrm{j})$ acidophil leukocyte $(\mathrm{k})$ basicyte

\section{Acknowledgments}

We express gratitude to Kejian Zhang of Hematological Department of Zhongnan Hospital, Wuhan University for providing us with bone marrow smears and giving us directions on medical issues. This work was supported by the Chinese National Ministry of Science and Technology under the grant 2001BA210A02

\section{References}

[1] Bennett, J.M., Catovsky, D., and Daniel, M.T., Proposals for the classification of the acute leukaemias. British Journal of Haematology, 1976. 33(4): p. 451-458.

[2] Park, J. and Keller, J.M., Snakes on the watershed. Ieee Transactions On Pattern Analysis And Machine Intelligence, 2001. 23(10): p. 1201-1205.

[3] Keller, J.M., Gader, P.D., et al. Soft counting networks for bone marrow differentials. 2001: Systems, Man, and Cybernetics, 2001 IEEE International Conference on.

[4] Jae-Sang, P. and Keller, J.M. Fuzzy patch label relaxation in bone marrow cell segmentation. in Systems, Man, and Cybernetics. 1997: IEEE International Conference on.

[5] Sobrevilla, P., Montseny, E., and Keller, J., White blood cell detection in bone marrow images, in 18th International Conference of the North American Fuzzy Information Processing Society - Nafips, R.N. Dave and T. Sudkamp, Editors. 1999, I E E E: New York. p. 403-407.

[6] Theera-Umpon, N., White blood cell segmentation and classification in microscopic bone marrow images, in Fuzzy Systems And Knowledge Discovery, Pt 2, Proceedings. 2005, Springer-Verlag Berlin: Berlin. p. 787-796.

[7] Nilsson, B. and Heyden, A., Segmentation of complex cell clusters in microscopic images: Application to bone marrow samples. Cytometry Part A, 2005. 66A(1): p. 24-31.

[8] Zhang, X.W., Song, J.Q., et al., Extraction of karyocytes and their components from microscopic bone marrow images based on regional color features. Pattern Recognition, 2004. 37(2): p. 351-361.

[9] Montseny, E., Sobrevilla, P., and Romani, S., A fuzzy approach to white blood cells segmentation in color bone marrow images, in 2004 Ieee International Conference on Fuzzy Systems, Vols 1-3, Proceedings. 2004, Ieee: New York. p. 173-178.

[10] Meschino, G.J. and Moler, E., Semiautomated image segmentation of bone marrow biopsies by texture features and mathematical morphology. Analytical And Quantitative Cytology And Histology, 2004. 26(1): p. 31-38.

[11] Hengen, H., Spoor, S., and Pandit, M. Analysis of blood and bone marrow smears using digital image processing techniques. 2002. San Diego, CA, United States: The International Society for Optical Engineering.

[12] Hardeberg, J.Y., Acquisition and Reproduction of Colour Images: Colorimetric and Multispectral approaches. 1999, Paris: Ecole Nationale Supérieure des Télécommunications.

[13] Tran, C.D., Development and analytical applications of multispectral imaging techniques: An overview. Fresenius Journal Of Analytical Chemistry, 2001. 369(3-4): p. 313-319.

[14] Morris, M.D., Microscopic and spectroscopic imaging of the chemical state. 1993, New York: Marcel Dekker.

[15] Levenson, R.M. and Hoyt, C.C., Spectral imaging and microscopy. American Laboratory, 2000. 32(22): p. 26-33.

[16] Turner, J.F., Chemical imaging comes of age. spie's oemagazine, 2002: p. 30-33.

[17] http://www.cri-inc.com.

[18] Gunn, S.R., Support Vector Machines for Classification and Regression. 1998, Faculty of Engineering and Applied 
Science, Department of Electronics and Computer Science, University of Southampton.

[19] Cortes, C. and Vapnik, V., Support-vector network. Machine Learning, 1995. 20: p. 273-297.

[20] Cristianini, N. and Shawe-Taylor, J., An Introduction to Support Vector Machines and Other Kernel-based Learning Methods. 2000, Cambridge: Cambridge University Press.

[21] Vapnik, V., Statistical Learning Theory. 1998, New York: Wiley.

[22] Friedman, J., Another approach to polychotomous classification. 1996, Department of Statistics, Stanford University.

[23] Hsu, C.W. and Lin, C.J., A simple decomposition method for support vector machines. Machine Learning, 2002. 46(1-3): p. 291-314.

[24] Lin, C.J., A Library for Support Vector Machines. 2003, http://www.csie.ntu.edu.tw/ cjlin/libsvm/index.html. 